\title{
Diseño de mezclas bituminosas para pavimentos con alquitrán, usando las metodologías Marshall y Ramcodes ${ }^{1}$
}

Recibido:

Mayo 29 de 2012

Aceptado:

Noviembre 10 de 2012

${ }^{1}$ Este articulo se origina en la investigación titulada "ANÁLISIS DE LA UTILIZACIÓN DE ESCORIA Y ALQUITRÁN

EN FABRICACIÓN DE MEZCLAS BITUMINOSAS PARA PAVIMENTOS",adscrita al Grupo de Investigación y desarrollo en Infraestructura Vial GRINFRAVIAL de la escuela de Ingeniería en Transporte y Vías de la Universidad Pedagógica y

Tecnólogica de Colombia. Esta

Investigación fue financiada por la Universidad Pedagógica y Tecnólogica de Colombia y con apoyo de la empresa Acerías Paz del Río S.A. en el marco de la investigación.

${ }^{2}$ Ingeniero en Transportes y Vías de la Universidad Pedagógica y Tecnológica de Colombia, Tunja (1997).

Especialista en Geotecnia Vial de la Universidad Pedagógica

y Tecnológica de Colombia, Sogamoso (2002). Candidato

a Magister en Ingeniería con énfasis en Infraestructura Vial

de la Universidad Pedagógica

y Tecnológica de Colombia,

Tunja. Docente del programa de Ingeniería de Transporte

y Vías e Investigador activo del grupo GRINFRAVIAL de

la Universidad Pedagógica y Tecnológica de Colombia, Tunja. Correo electrónico: ricardo.ochoa@uptc.edu.co

\section{Resumen}

El presente documento plantea una alternativa de utilización del alquitrán de hulla, subproducto de la industria siderúrgica, dado el problema de su acumulación e impacto ambiental negativo. Por lo anterior, se observa la necesidad de analizar la incorporación de alquitrán de hulla como ligante en mezclas para pavimentos. De este modo, inicialmente se presenta la procedencia, descripción de las principales características y propiedades del alquitrán. Seguidamente, se formula y evalúa la mezcla, mediante el procedimiento de las metodologías Marshall y RAMCODES, para determinar su resistencia. Con los resultados obtenidos de los ensayos se obtuvieron las propiedades físicas y mecánicas de la mezcla. Teniendo en cuenta los resultados de las dos metodologías se realiza una comparación para establecer la conveniencia de utilizar la metodología RAMCODES en el diseño de mezclas. Por último, se analiza la alternativa de utilizar el alquitrán de hulla como ligante en mezclas bituminosas para pavimentos.

Palabras clave: Alquitrán de hulla, mezclas bituminosas, Marshall, RAMCODES.

\section{Abstract}

This paper presents an alternative use of coal tar, a byproduct of the steel industry, given the problem of accumulation and environmental impact negative. Therefore, there is a need to analyze the incorporation of coal tar as a binder in paving mixtures. Thus, this paper presents initially the origin, description of the main characteristics and properties of tar. Later, the mix is formulated and evaluated, by means of the procedure of RAMCODES and Marshall methodologies, to determine its resistance. With the results of the tests are obtained physical and mechanical properties of the mix. Taking into account the results of both methods, it makes a comparison to determine the suitability to use the methodology RAMCODES in the mix design. Finally, it analyzes the alternative to use coal tar as binder in bituminous mix for pavement.

Keywords: Coal tar, road surface mixtures, Marshall, RAMCODES. 


\section{Introducción}

n Colombia la infraestructura vial
no ha evolucionado como todos
esperábamos. La red vial se ha encontrado ante varios retos, en especial lo concerniente a los altos costos de las mezclas asfálticas utilizadas como capa de rodadura. Lo anterior, en gran parte obedece a los altos precios internacionales del petróleo, lo cual se refleja automáticamente en el precio del asfalto.

El actual costo de las mezclas asfálticas ha limitado el desarrollo de la infraestructura vial del país, especialmente en lo que respecta a vías de segundo orden. La red de carreteras del país es de alrededor $164.000 \mathrm{Km}$, de los cuales un $15 \%$ se encuentra pavimentado. De los $164.000 \mathrm{Km}, 16.776 \mathrm{Km}$ son de red primaria y $147.500 \mathrm{Km}$ de red secundaria y terciaria. $13.296 \mathrm{Km}$ de red primaria están a cargo del Instituto Nacional de Vías (INVIAS) y $3.380 \mathrm{Km}$ son concesionados. Por su parte, la red secundaria y terciaria se encuentran distribuidas así: $72.761 \mathrm{Km}$ a cargo de los departamentos, $4.918 \mathrm{Km}$ a cargo de los municipios, $27.577 \mathrm{Km}$ responsabilidad del Instituto Nacional de Vías y $12.251 \mathrm{Km}$ de los privados [1].

Ante la situación anterior, surge la necesidad de buscar alternativas en la posibilidad de utilizar otros productos, que en ningún momento pretenden reemplazar el asfalto como ligante, como es el caso del alquitrán para la elaboración de mezclas bituminosas que puedan utilizarse como capas de rodadura en vías secundarias y terciarias.

Para utilizar el alquitrán como ligante es imperioso conocer sus características físicas y químicas, para lo cual se consultó en el laboratorio de Acerías Paz del Río S.A. lo relacionado con las propiedades químicas. Para la obtención de las propiedades físicas se realizaron pruebas de laboratorio enmarcadas dentro de las normas de ensayos de materiales para carreteras del INVIAS [2], así como la caracterización de los agregados. Para el diseño de las mezclas se trabajó con las metodologías Marshall y RAMCODES, con el objeto de realizar una comparación y evaluación de la conveniencia de utilizar esta nueva metodología (RAMCODES), para la obtención del contenido óptimo de ligante y de las propiedades de la mezcla.

Este trabajo pretende contribuir al desarrollo en la utilización del alquitrán, un subproducto de la industria siderúrgica, y mejorar las condiciones ambientales en sus zonas de influencia mediante la disminución de la acumulación del alquitrán, que causa afectación ambiental en el entorno.

\section{Materiales y métodos}

\section{Alquitrán como ligante}

El alquitrán, como se observa en la figura1, es un producto bituminoso semisólido o líquido que se obtiene como residuo de la destilación, en ausencia de aire, de sustancias orgánicas que posean materiales volátiles, como carbón o madera. Es un ligante en frío, resistente al agua, insoluble en aceites lubricantes y combustibles derivados del petróleo, soluble en éter, benceno, sulfuro de carbono, cloroformo y quinoleína. El alquitrán está formado por componentes alifáticos y naftalénicos que contienen hidrógeno, oxígeno, nitrógeno y azufre [3].

El alquitrán utilizado en este estudio es producido en la planta de Coquización, cuyo proceso se realiza a $1000^{\circ} \mathrm{C}$ [4]. En la tabla 1 se muestra las propiedades físico-químicas de este alquitrán.

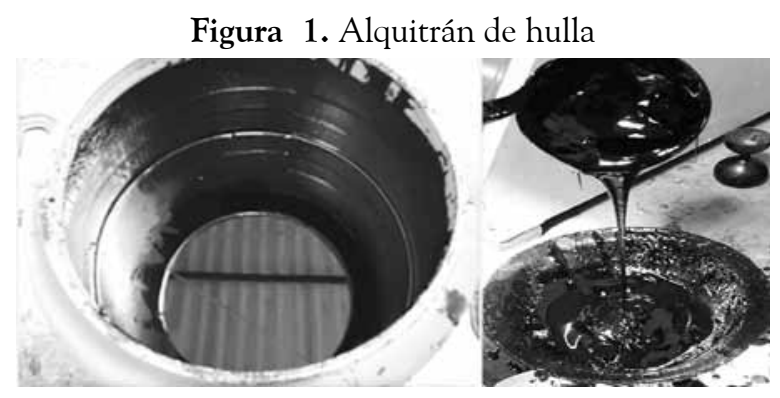


Diseño de mezclas bituminosas para pavimentos con alquitrán,

Tabla 1. Propiedades físico-químicas del alquitrán

\begin{tabular}{|c|c|c|}
\hline PARAMETRO & UNIDAD & PROMEDIO \\
\hline Humedad & $\%$ & 7 \\
\hline Cenizas & $\%$ & 0.10 \\
\hline $\begin{array}{c}\text { Insolubles en } \\
\text { Quinoleína }\end{array}$ & $\%$ & 4 \\
\hline Insolubles en Tolueno & $\%$ & 3 \\
\hline Poder calorífico & $\mathrm{kcal} / \mathrm{kg}$ & 8900 \\
\hline
\end{tabular}

Fuente: Departamento Laboratorio Acerías Paz del Río S.A.

Las propiedades físicas se determinaron en laboratorio simulando éste a un asfalto líquido, los ensayos realizados fueron: ensayo de viscosidad Saybolt-Furol, para determinar el grado de fluidez del alquitrán; ensayo de Punto de Ignición y Llama, para determinar la temperatura a la cual los vapores del alquitrán se encienden al ser sometidos a calentamiento en presencia de una pequeña llama, así como determinar la temperatura a la cual la presencia de la llama produce el encendido del alquitrán y ensayo de gravedad específica.

\section{Agregados pétreos}

Los materiales utilizados en una mezcla bituminosa deben ser sometidos a rigurosos estudios en los cuales se establece la posibilidad de su utilización. Para esto, como agregado grueso se utilizó material triturado tipo grava, el cual se sometió a los siguientes ensayos: desgaste en la máquina de los ángeles, porcentaje de caras fracturadas, índice de alargamiento e índice de aplanamiento.

Además se realizaron ensayos para determinar la gravedad específica y el porcentaje de absorción. Como agregado fino se utilizó arena y como llenante mineral se utilizó cemento Portland y finos de arena pasa tamiz \#200, a los cuales se determinaron sus gravedades específicas. Todos los ensayos se hicieron teniendo en cuenta las normas de ensayo para materiales de carretera del INVÍAS [2].
El tipo de mezcla escogido para la realización de este estudio fue una mezcla densa en caliente tipo 2 (MDC-2) de acuerdo al Artículo 450-07 de las especificaciones generales de construcción de carreteras del INVÍAS [5].

La metodología utilizada para el desarrollo de esta investigación se planteó en tres etapas: la primera la realización de todos los ensayos para conocer las características de los materiales a utilizar en el diseño de las mezclas bituminosas, la segunda fue la realización de los ensayos necesarios para la obtención de la fórmula de trabajo de la mezcla bituminosa por la metodología Marshall y por RAMCODES, y por último la comparación de los resultados de las dos metodologías y el análisis de la conveniencia de la utilización del alquitrán.

\section{Método Marshall}

El propósito de este método es determinar el contenido óptimo de ligante para una mezcla específica de agregados; así como también proporcionar información sobre las características físicas y mecánicas de la mezcla bituminosa en caliente, de tal manera que sea posible establecer si cumplen los parámetros de densidades y contenidos óptimos de vacío durante la construcción de la capa de pavimento.

El objetivo general del procedimiento de diseño consiste en determinar una combinación y gradación económica de agregados y ligante que produzca una mezcla con: suficiente ligante para garantizar un pavimento durable, adecuada estabilidad para que satisfaga las demandas de tránsito sin producir deformación o desplazamiento, un contenido de vacíos lo suficientemente alto para permitir una ligera cantidad de compactación adicional bajo las cargas de tránsito, sin que se produzca exudación o pérdida de estabilidad y todavía lo suficientemente bajo para no dejar penetrar los efectos dañinos del aire y el agua, suficiente trabajabilidad para permitir una colocación eficiente sin segregación.
Diciembre 2012 ISSN 0122-820X 


\section{Método RAMCODES}

El método RAMCODES [6], acrónimo de Rational Methodology for Compacted Geomaterial's Densification and Strenght Analysis desarrollado por FJ. Sánchez-Leal, es una metodología basada en experimentos factoriales y en la experiencia práctica de diseño y control, para análisis de densificación y resistencia de geomateriales compactados.

El Marshall acelerado por RAMCODES es una aplicación elaborada para el diseño rápido del procedimiento Marshall tradicional, el cual liga racionalmente las especificaciones de diseño con los criterios de control en campo, mediante la implementación del "polígono de vacíos" que define un área donde se cumplen todas las especificaciones de vacíos (VAM, VAF y VA). Los vacíos están en función del contenido de ligante (\%CA) y densidad Bulk (Gmb) y se representan en mapas como isolíneas, para los valores permitidos, la intersección de estas líneas produce una construcción gráfica en el espacio \%CA - Gmb, que da lugar al polígono el cual por medio de su centroide es posible matemáticamente la obtención de un contenido de asfalto que cumplan a la vez todas las especificaciones de vacíos de la mezcla [7]

Los siguientes pasos han sido propuestos para modificar o acelerar el procedimiento de diseño Marshall original [8]:

- Determine la gravedad específica efectiva (Gse), la gravedad específica Bulk de la combinación de agregados (Gsb), y la gravedad específica aparente (Gsa) de la combinación de agregados seleccionada. Verifique que Gsa $>$ Gse $>$ Gsb según las definiciones teóricas. Si no verifica, revise los ensayos correspondientes.

- Obtenga matemáticamente, del polígono de vacíos, el contenido óptimo de ligante teniendo en cuenta las especificaciones y las gravedades específicas de la combinación de agregados. Para determinar el centroide del polígono de vacíos, estos pueden ser fácilmente automatizados en una hoja electrónica convencional excel o bien, se puede utilizar RAMSOFT.

- Siguiendo las disposiciones del ensayo Marshall, mezcle la combinación de agregados con el contenido óptimo de ligante y compacte tres especímenes bajo la energía de compactación seleccionada. Mida los especímenes para determinar los vacíos, y ensáyelos para determinar la estabilidad y el flujo. Promedie los resultados.

-Verifique si el promedio del contenido de ligante y la densidad Bulk entra en el polígono de vacíos. Si no verifica, entonces el intento falló. Seleccione otra combinación de agregados y vaya al primer paso.

-Verifique si el promedio de estabilidad y flujo de los especímenes cumplen con las especificaciones. Si no verifica, entonces el intento falló. Seleccione otra combinación de agregados y vaya al primer paso.

\section{Resultados}

\section{Caracterización de los materiales}

De acuerdo a la metodología propuesta, a continuación se presentan los resultados de los ensayos realizados a los materiales usados en la investigación. La tabla 2, presenta la caracterización del ligante utilizado (Alquitrán de Hulla).

Tabla 2. Caracterización del alquitrán de hulla

\begin{tabular}{|c|c|c|}
\hline NORMA & ENSAYO & RESULTADO \\
\hline INV E-707-07 & $\begin{array}{c}\text { Gravedad específica de } \\
\text { materiales bituminosos } \\
\text { (Método del picnómetro) }\end{array}$ & 1.12 \\
\hline INV E-709-07 & $\begin{array}{c}\text { Punto de ignición } \\
\text { mediante la copa abierta } \\
\text { de Cleveland }\end{array}$ & $80^{\circ} \mathrm{C}$ \\
\hline INV E-709-07 & $\begin{array}{c}\text { Punto de llama mediante } \\
\text { la copa abierta de } \\
\text { Cleveland }\end{array}$ & $200 \mathrm{~s}$ \\
\hline INV E 714-07 & Viscosidad Saybolt Furol & $2.2 \%$ \\
\hline INV E-723-07 & $\begin{array}{c}\text { Destilación de ligantes } \\
\text { líquidos }\end{array}$ \\
\hline
\end{tabular}

En la tabla 3, se presentan los resultados de los ensayos realizados al agregado grueso. En las tablas 4 y 5 , se muestran los resultados de la caracterización de la arena 
utilizada como agregado fino y la densidad del cemento Portland y la arena fina pasa tamiz \#200, utilizados como llenante mineral, respectivamente. Al comparar estos resultados con las especificaciones generales de construcción de carreteras del INVIAS 2007 [5], los materiales pueden ser utilizados para la fabricación de mezclas bituminosas para pavimentos.

\section{Diseño experimental}

En la tabla 6, se muestran las seis (6) distribuciones granulométricas con sus respectivas dosificaciones, la variable es el tipo de llenante mineral (cemento o arena), ajustadas a la franja granulométrica de una mezcla tipo MDC-2, escogida para el proceso en los ensayos de laboratorio para las metodologías Marshall y RAMCODES, basando su desarrollo en dos etapas.

Las granulometrías se ajustaron para que dentro de la franja de una mezcla MDC-2, estas estuvieran justo en el centro, por el lado fino y por el lado grueso de dicha franja, figura 2 , esto con el objeto de evaluar la incidencia de la granulometría en el comportamiento de la mezcla.

Tabla 3. Caracterización del agregado grueso

\begin{tabular}{|c|c|c|c|}
\hline NORMA & ENSAYO & $\begin{array}{l}\text { RESUL } \\
\text { TADO }\end{array}$ & $\begin{array}{l}\text { ESPECI- } \\
\text { FICACIÓN }\end{array}$ \\
\hline INV E-218-07 & $\begin{array}{l}\text { Desgaste en la } \\
\text { máquina de los } \\
\text { ángeles }\end{array}$ & $16.64 \%$ & 25\% máx. \\
\hline INV E-227-07 & $\begin{array}{l}\text { Porcentaje de caras } \\
\text { fracturadas }\end{array}$ & $100 \%$ & 60\% mín. \\
\hline INV E-230-07 & $\begin{array}{c}\text { Índice de } \\
\text { alargamiento }\end{array}$ & $17.62 \%$ & - \\
\hline INV E-230-07 & $\begin{array}{c}\text { Índice de } \\
\text { aplanamiento }\end{array}$ & $23.92 \%$ & - \\
\hline \multirow{2}{*}{ INV E-223-07 } & \multirow{2}{*}{$\begin{array}{l}\text { Gravedad especifica } \\
\text { bulk (Gsb) y absorción }\end{array}$} & $\begin{array}{l}\text { Gravedad } \\
\text { específica }\end{array}$ & Absorción \\
\hline & & 2.44 & \multirow[b]{3}{*}{$2.32 \%$} \\
\hline INV E-223-07 & $\begin{array}{l}\text { Gravedad especifica } \\
\text { (Gsb-sss) y absorción }\end{array}$ & 2.50 & \\
\hline INV E-223-07 & $\begin{array}{l}\text { Gravedad especifica } \\
\text { aparente (Gsa) y } \\
\text { absorción }\end{array}$ & 2.59 & \\
\hline
\end{tabular}

Tabla 4. Caracterización del agregado fino

\begin{tabular}{|c|c|c|c|}
\hline NORMA & ENSAYO & $\begin{array}{c}\text { RESUL- } \\
\text { TADO }\end{array}$ & $\begin{array}{c}\text { ESPECI- } \\
\text { FICACIÓN }\end{array}$ \\
\hline INV E-133-07 & $\begin{array}{c}\text { Equivalente de } \\
\text { arena }\end{array}$ & $70 \%$ & $50 \%$ mín. \\
\hline INV E-222-07 & $\begin{array}{c}\text { Gravedad } \\
\text { especifica } \\
\text { bulk (Gsb) y } \\
\text { absorción }\end{array}$ & $\begin{array}{c}\text { Gravedad } \\
\text { específica }\end{array}$ & Absorción \\
\cline { 1 - 3 } INV E-222-07 & $\begin{array}{c}\text { Gravedad } \\
\text { especifica } \\
\text { (Gsb-sss) y } \\
\text { absorción }\end{array}$ & 2.23 & \multirow{2}{*}{$1.36 \%$} \\
\cline { 1 - 3 } INV E-222-07 & $\begin{array}{c}\text { Gravedad } \\
\text { especifica } \\
\text { aparente (Gsa) } \\
\text { y absorción }\end{array}$ & \multicolumn{2}{|c}{} \\
\hline
\end{tabular}

Tabla 5. Propiedades del llenante mineral

\begin{tabular}{|c|c|c|c|}
\hline MATERIAL & NORMA & ENSAYO & $\begin{array}{c}\text { RESUL- } \\
\text { TADO }\end{array}$ \\
\hline $\begin{array}{c}\text { CEMENTO } \\
\text { PORTLAND }\end{array}$ & INV E-307-07 & $\begin{array}{c}\text { Densidad del } \\
\text { cemento }\end{array}$ & 3.10 \\
\hline $\begin{array}{c}\text { ARENA PASA } \\
\text { TAMIZ No 200 }\end{array}$ & INV E-222-07 & $\begin{array}{c}\text { Gravedad } \\
\text { específica efectiva }\end{array}$ & 2.74 \\
\hline
\end{tabular}

En la primera etapa, se realizaron seis diseños Marshall para el desarrollo experimental, tres con cemento como llenante y tres con arena pasa tamiz \#200, la temperatura de calentamiento de los agregados y el ligante fue de $40^{\circ} \mathrm{C}$ y con una compactación constante de 75 golpes por cara (nivel de tránsito NT-2), realizando un experimento factorial variando dos factores, el contenido de ligante variable en $0.5 \%$ para cada mezcla y la gradación variable de acuerdo a la ubicación en la franja granulométrica. Lo anterior, con el objeto de encontrar la fórmula de trabajo para el diseño Marshall, seguido del proceso de compactación y toma de resultados del comportamiento mecánico de las mezclas.

\begin{tabular}{|c|c|c|c|}
\hline ERIAL & NORMA & ENSAYO & $\begin{array}{c}\boldsymbol{R E S U L}- \\
\boldsymbol{T A D O}\end{array}$ \\
\hline IENTO & INV E-307-07 & $\begin{array}{c}\text { Densidad del } \\
\text { cemento }\end{array}$ & 3.10 \\
LAND & & $\begin{array}{c}\text { Gravedad } \\
\text { específica efectiva }\end{array}$ & 2.74 \\
\hline
\end{tabular}

ISSN 0122-820X 


\section{Respuestas}

Año 17

No. 2

Diciembre 2012 ISSN 0122-820X compactación y toma de resultados de estabilidad y flujo de las mezclas.

Tabla 6. Distribuciones granulométricas y tipo de agregados de las mezclas

\begin{tabular}{|c|c|c|c|c|}
\hline MEZCLA & AGREGADO & MATERIAL & $\begin{array}{l}\text { PORCEN. } \\
\text { TAJE }\end{array}$ & $\begin{array}{l}\text { DISTRIBU. } \\
\text { CIÓN }\end{array}$ \\
\hline \multirow{3}{*}{ M-1 } & Grueso & Grava & 41 & \multirow{3}{*}{$\begin{array}{l}\text { Lado grueso } \\
\text { de la franja } \\
\text { granulométrica }\end{array}$} \\
\hline & Fino & Arena & 52 & \\
\hline & Llenante & Cemento & 7 & \\
\hline \multirow{3}{*}{$M-2$} & Grueso & Grava & 46 & \multirow{3}{*}{$\begin{array}{c}\text { Centro de } \\
\text { la franja } \\
\text { granulométrica }\end{array}$} \\
\hline & Fino & Arena & 49 & \\
\hline & Llenante & Cemento & 5 & \\
\hline \multirow{3}{*}{$M-3$} & Grueso & Grava & 50 & \multirow{3}{*}{$\begin{array}{c}\text { Lado fino } \\
\text { de la franja } \\
\text { granulométrica }\end{array}$} \\
\hline & Fino & Arena & 46 & \\
\hline & Llenante & Cemento & 4 & \\
\hline \multirow{3}{*}{ M-4 } & Grueso & Grava & 40 & \multirow{3}{*}{$\begin{array}{l}\text { Lado grueso } \\
\text { de la franja } \\
\text { granulométrica }\end{array}$} \\
\hline & Fino & Arena & 54 & \\
\hline & Llenante & $\begin{array}{l}\text { Arena pasa } \\
\quad \# 200\end{array}$ & 6 & \\
\hline \multirow{3}{*}{ M-5 } & Grueso & Grava & 44 & \multirow{3}{*}{$\begin{array}{c}\text { Centro de } \\
\text { la franja } \\
\text { granulométrica }\end{array}$} \\
\hline & Fino & Arena & 51 & \\
\hline & Llenante & $\begin{array}{l}\text { Arena pasa } \\
\quad \# 200\end{array}$ & 5 & \\
\hline \multirow{3}{*}{ M-6 } & Grueso & Grava & 47 & \multirow{3}{*}{$\begin{array}{c}\text { Lado fino } \\
\text { de la franja } \\
\text { granulométrica }\end{array}$} \\
\hline & Fino & Arena & 49 & \\
\hline & Llenante & $\begin{array}{l}\text { Arena pasa } \\
\text { \#200 }\end{array}$ & 4 & \\
\hline
\end{tabular}

Una vez compactadas las probetas, se dejaron enfriar durante 15 minutos, antes de sacarlas del molde. Una vez fuera del molde se dejaron enfriar y curar a temperatura ambiente por ocho días, de tal manera que se liberen los solventes de la mezcla. Posterior a este tiempo, se continuó con la realización del ensayo y la determinación de la estabilidad y flujo en la prensa Marshall.

Resultados de las pruebas realizadas con las dos metodologías

A continuación, en las tablas 7 y 8, se presentan los resultados obtenidos de las mezclas diseñadas mediante las dos metodologías.

Basados en el hecho de que la metodología RAMCODES utiliza parámetros y fórmulas del diseño Marshall, se espera obtener resultados similares. Esto se puede constatar con los resultados obtenidos en este proyecto, donde se consiguieron respuestas muy parecidas empleando las dos metodologías con relación a las propiedades volumétricas y mecánicas de cada una de las mezclas.

Según los resultados obtenidos y teniendo en cuenta las especificaciones de la tabla 450.4 del artículo 450-07 de las especificaciones generales de construcción de carreteras del INVIAS [5], las mezclas preparadas con cemento portland como llenante cumplen para un nivel de tránsito NT-1, sin embargo el flujo está un poco por encima del rango de 2-4 mm. En los resultados observados de las mezclas preparadas con arena pasa tamiz \#200 como llenante, algunos parámetros cumplen las especificaciones para un nivel de tránsito NT-1, aunque el flujo si resultó muy alejado del rango especificado. Los resultados de la metodología Marshall para la mezcla $\mathrm{M}-2$,

Figura 2. Distribución granulométrica de las mezclas

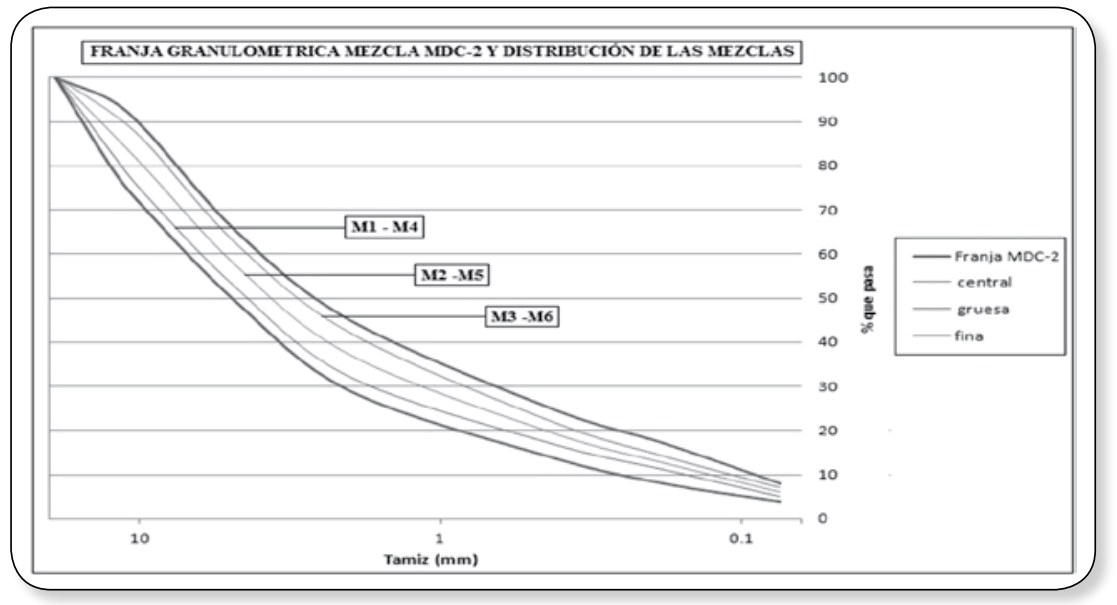


Diseño de mezclas bituminosas para pavimentos con alquitrán,

cumplen con todas las especificaciones para un nivel de tránsito NT-2, siendo así está la fórmula de trabajo que mejor comportamiento mecánico presenta.

Tabla 7. Resultados de las mezclas diseñadas con cemento como llenante

\begin{tabular}{|c|c|c|c|c|c|c|}
\hline \multicolumn{7}{|c|}{ MEZCLA DISEÑADAS CON GRAVA, ARENA Y CEMENTO } \\
\hline \multirow[b]{2}{*}{$\begin{array}{l}\text { PARÁ- } \\
\text { METRO }\end{array}$} & \multicolumn{2}{|c|}{ M-1 } & \multicolumn{2}{|c|}{ M-2 } & \multicolumn{2}{|c|}{ M-3 } \\
\hline & $\begin{array}{l}\text { Mar- } \\
\text { shall }\end{array}$ & $\begin{array}{c}\text { RAM- } \\
\text { CODES }\end{array}$ & $\begin{array}{l}\text { Mar- } \\
\text { shall }\end{array}$ & $\begin{array}{l}\text { RAM- } \\
\text { CODES }\end{array}$ & $\begin{array}{l}\text { Mar- } \\
\text { shall }\end{array}$ & $\begin{array}{l}\text { RAM- } \\
\text { CODES }\end{array}$ \\
\hline Alquitrán (\%) & 7.40 & 7.13 & 6.50 & 6.87 & 6.42 & 6.63 \\
\hline $\mathrm{Gmb}\left(\mathrm{gr} / \mathrm{cm}^{3}\right)$ & 2.13 & 2.14 & 2.14 & 2.13 & 2.13 & 2.13 \\
\hline $\begin{array}{l}\text { Estabilidad } \\
(\mathrm{Kg})\end{array}$ & 734 & 793 & 792 & 755 & 668 & 688 \\
\hline Flujo $(\mathrm{mm})$ & 4.13 & 4.03 & 3.88 & 4.13 & 4.26 & 4.22 \\
\hline $\mathrm{Vv}_{\mathrm{v}}(\%)$ & 4.0 & 3.86 & 4.0 & 3.88 & 4.0 & 3.88 \\
\hline VAM (\%) & 16.37 & 15.78 & 15.22 & 15.80 & 15.53 & 15.81 \\
\hline VFA (\%) & 75.70 & 75.51 & 73.90 & 75.43 & 74.30 & 75.43 \\
\hline
\end{tabular}

Tabla 8. Resultados de las mezclas diseñadas con arena como llenante

\begin{tabular}{|c|c|c|c|c|c|c|}
\hline \multicolumn{7}{|c|}{$\begin{array}{l}\text { MEZCLA DISEÑADAS CON GRAVA, ARENA Y ARENA PASA } \\
\text { TAMIZ \# } 200\end{array}$} \\
\hline \multirow[b]{2}{*}{$\begin{array}{l}\text { PARA- } \\
\text { METRO }\end{array}$} & \multicolumn{2}{|c|}{ M-4 } & \multicolumn{2}{|c|}{ M-5 } & \multicolumn{2}{|c|}{ M-6 } \\
\hline & $\begin{array}{l}\text { Mar- } \\
\text { shall }\end{array}$ & RAM- & $\begin{array}{l}\text { Mar- } \\
\text { shall }\end{array}$ & $\begin{array}{l}\text { RAM- } \\
\text { CODES }\end{array}$ & $\begin{array}{l}\text { Mar- } \\
\text { shall }\end{array}$ & $\begin{array}{l}\text { RAM- } \\
\text { CODES }\end{array}$ \\
\hline Alquitrán (\%) & 6.60 & 6.80 & 6.60 & 6.70 & 6.20 & 6.70 \\
\hline $\mathrm{Gmb}\left(\mathrm{gr} / \mathrm{cm}^{3}\right)$ & 2.18 & 2.18 & 2.17 & 2.17 & 2.18 & 2.17 \\
\hline $\begin{array}{l}\text { Estabilidad } \\
\text { (Kg) }\end{array}$ & 527 & 472 & 702 & 643 & 665 & 588 \\
\hline Flujo (mm) & 8.0 & 10.1 & 9.2 & 10.0 & 8.6 & 9.8 \\
\hline $\mathrm{V}_{\mathrm{v}}(\%)$ & 4.0 & 3.9 & 4.0 & 3.9 & 4.0 & 3.9 \\
\hline VAM (\%) & 15.6 & 15.8 & 15.8 & 15.8 & 15.0 & 15.8 \\
\hline VFA (\%) & 73.7 & 75.5 & 74.0 & 75.5 & 73.0 & 75.5 \\
\hline
\end{tabular}

\section{Conclusiones}

Este trabajo pretende abrir la investigación dirigida a evaluar el comportamiento de mezclas bituminosas para pavimentos utilizando otro tipo de ligante diferente al asfalto.

No se pretende reemplazar el asfalto en la elaboración de mezclas para pavimentos, la intensión es buscar alternativas que permitan escoger el producto que más se ajuste a las necesidades de cada proyecto específico.
RAMCODES es una metodología de gran utilidad para el diseño, producción y control de calidad de mezclas bituminosas, puesto que garantiza el cumplimiento de los parámetros volumétricos exigidos por las especificaciones. Además, existen varios beneficios al utilizar esta metodología como ahorro de tiempo, recursos y dinero, gracias a que solo es necesario realizar tres briquetas en comparación con 15 briquetas de la metodología Marshall.

Entre las observaciones encontradas en el desarrollo de esta investigación, RAMCODES demostró, por medio de sus aplicaciones, ser una herramienta útil en la interpretación de las respuestas de los materiales compactados basados en el comportamiento de vacíos y las trayectorias del incremento de ligante en la mezcla.

De acuerdo con las metodologías, se puede decir que la mezcla M-2 va a presentar un mejor comportamiento a lo largo de su vida útil, debido a que tiene una relación muy cercana con las especificaciones de construcción de carreteras del INVIAS-2007 para niveles de tránsito NT-1 y NT-2.

La incorporación del cemento como llenante jugó un papel importante en el comportamiento de la mezcla debido a que mejoró las propiedades del ligante, mejorando la adherencia con los agregados permitiendo mejores respuestas de estabilidad y flujo.

Sin pretender desplazar el uso de asfalto en la fabricación de mezclas para pavimentos, si se puede pensar en la utilización de alquitrán de hulla en la fabricación de estas mezclas para ser utilizadas en vías secundaria y terciarias, encontrando un comportamiento aceptable siempre y cuando se cumplan todos los requerimientos para poder cumplir con las especificaciones.
Diciembre 2012 ISSN 0122-820X 


\section{Referencias bibliográficas}

[1] MINISTERIO DE TRANSPORTE. (2008). Oficina Asesora de Planeación. Diagnóstico del sector Transporte, 2008, p. 47.

[2] INSTITUTO NACIONAL DE VÍAS (INVIAS), (2007). Normas de Ensayos de Materiales para Carreteras. V. I y II. Bogotá D.C.

[3] Acerías Paz del Río S.A. Disponible en $\quad<$ http://www.pazdelrio.com.co/ alquitran.htm/>. Fecha de consulta 25 de enero de 2012.

[4] Gómez, Alfonso, (2002). Proceso siderúrgico Planta Belencito. Acerías Paz del Río S.A.

[5] INSTITUTO NACIONAL DE VÍAS (INVIAS), (2007). Especificaciones generales de construcción de carreteras. Bogotá D. C.

[6] Sánchez Leal, Freddy J, RAMCODES: Metodología racional para el análisis de densificación y resistencia de geomateriales compactados. Descripción de la metodología y campo de aplicación. Publicación técnica 200 ed. Sanfandila, Querétaro, México. 2002. ISSN 0188-7297.

[7] DELGADO AlAmilla, Horacio, et al. Influencia de la granulometría en las propiedades volumétricas de la mezcla asfáltica. Metodología RAMCODES, RAMCODES en las mezclas asfálticas. Publicación Técnica 299 ed. Sanfandila, Querétaro, México. 2006. ISSN 01887297. Citado SÁNCHEZ-LEAL, F. J. Manual de aplicación RAMCODES. Venezuela: Solestudios C.A. 2008. usando las metodologías Marshall y Ramcodes

[8] SÁNCHEZ-LEAL, F. J. Manual de aplicación RAMCODES. Venezuela: Solestudios C.A. 2008. 